\title{
Antiepileptics and Sound Perception Disorders
}

\section{ASHWANI BHASKER}

Epilepsy, a neurological health issue has been reported in all age groups and all countries. Anti-epileptic drugs have been reported to have many adverse events affecting different body parts and organs but their hazardous affect on a primary sensory organ is less known. In this mini review, we discuss thee impact of anti-epileptic medications on sound perception which adversely disrupts the quality of life.

KEYWORDS: Epilepsy, Sound, Perception

\section{INTRODUCTION}

Epilepsy is a neurological disorder affecting approximately $1 \%$ of the world's population. ${ }^{1}$ Epileptic seizures result in a set of brutal, unpredictable, and transient clinical manifestations resulting from the paroxysmal hyperactivity of a hyperexcitable neural network and its possible spread. Epilepsy treatment is based primarily on pharmacological treatment to reduce or even suppress the frequency of seizures and the pharmacodynamics of antiepileptic drugs vary according to the molecules, inducing one or more of these mechanisms: a strengthening of synaptic inhibition, a decrease in synaptic arousal, or even stabilization of cell membranes.

Because of their specific mechanism of action, antiepileptic drugs have indications in the treatment of one or more forms of epilepsy. Carbamazepine, sodium valproate, lamotrigine, levetiracetam can be used in several indications, such as generalized seizures tonic clonic seizures, absence seizures, myoclonia or partial seizures. Apart from epilepsy, certain molecules are also indicated in the treatment of bipolar syndromes, neuropathic pain, or certain facial neuralgia.

From a safety perspective, antiepileptics have a pattern of adverse events that vary from molecule to molecule, which may lead the clinician to reassess the patient's individual benefit/risk ratio. Among the most wellknown examples, particularly due to their severity, are Stevens-Johnson syndromes due to lamotrigine ${ }^{2}$, hyponatremias with carbamazepine and oxcarbazepine ${ }^{3}$, gingival hypertrophies under phenytoin ${ }^{4}$ or fetal malformations following sodium valproate. 5 In addition, pharmacological therapeutic follow-up may be performed at the individual level due to a narrow therapeutic margin and/or the risk of drug interactions for certain molecules. ${ }^{6-8}$

Because of their diffusion to the central nervous system, antiepileptic drugs expose patients to neurological adverse effects. Cognitive disorders under phenobarbital and aggressive states under lacosamide, felbamate, lamotrigine or levetiracetam are fairly well described examples, especially in the paediatric population. ${ }^{9}$ Hamed SA in a review reported that longterm use of certain antiepileptic drugs was associated with the rare occurrence of tinnitus, hearing loss, or dizziness, including therapeutic doses. Although mostly reversible, these audio-vestibular abnormalities may persist despite discontinuation of treatment. ${ }^{10}$ These hearing disorders appear to be less present in the collective consciousness of health professionals, potentially explained by the rarity of these adverse effects and non-serious symptomatology.

In the literature, the majority of hearing disorders in the perception of antiepileptic sounds concern carbamazepine. Tateno et al. published a case series of six cases of change in tone perception under carbamazepine and identified a few previously published cases. ${ }^{13}$ The time frames for appearance, when specified, ranged from a few hours to two weeks after the introduction of CBZ. For all reported cases, the patients were all musicians, often possessing the absolute ear, 
and probably more likely to notice moderate variations in tone perception than other patients treated with CBZ. The dosages used ranged from 100 to 6oomg/day. Threequarters were treated for epilepsy (with no particular profile reported), the rest for psychiatric disorders or neuropathic pain." In the majority of patients, the adverse reaction rapidly regressed to the discontinuation of the offending drug or the decrease in dosage. Some patients, however, appeared to see a spontaneous improvement in symptoms despite continued treatment at the same dose.

Another case of hearing impairment in an epileptic subject has been reported with anti-epileptic drugs as causative agents. ${ }^{12}$ whose symptoms disappeared despite the continuation of treatment at the same dosage. A genetic predisposition can be evoked but so far none of the authors who reported the cases have discussed this point.

Till date, carbamazepine, oxcarbazepine and lacosamide have been reported in the literature to be associated with the occurrence of sound perception disorders. Their mechanism of action could be the common feature of this pathophysiology, exceeding the hypothesis of a structural-toxicity relationship, since these are different. The mechanisms involved in the occurrence of these tonal perception anomalies are not elucidated for any of these molecules. However, several hypotheses have been raised, such as the possibility of a direct effect on the central nervous system. Indeed, interference with the sodium channels would alter the action potential of the auditory nerves and cochlear nuclei of the brainstem [10]. Furthermore, hypotheses of local action on the auditory system, with modification of the mechanical properties of the hair cells of Corti's organ, or an action on the sarcolemma of the ear muscle, affecting the tension of the tympanic membrane..$^{11-14}$

Changing the perception of sounds is a very rare adverse effect of carbamazepine, oxcarbazepine and lacosamide, identified in the literature. Rapid onset after the introduction of treatment, a complete resolution of symptoms, in most cases at the end of treatment, is observed, without any after-effects appear to be reported. Because of the impact on quality of life due to impairment of a basic sensory organ, knowledge of this adverse effect seems important to evoke this diagnosis.

\section{REFERENCES}

1. Sankaraneni R, Lachhwani D. Antiepileptic drugs - A review. Pediatr Ann 2015;44(2):e36-42.

2. Borrelli EP, Lee EY, Descoteaux AM, Kogut SJ, Caffrey AR. Stevens-Johnson syndrome and toxic epidermal necrolysis with antiepileptic drugs: an analysis of the US Food and Drug Administration Adverse Event Reporting System. Epilepsia 2018;59(12):2318-24.

3. Berghuis B, van der Palen J, de Haan GJ, Lindhout D, Koeleman BPC, Sander JW, et al. Carbamazepine- and oxcarbazepine- induced hyponatremia in people with epilepsy. Epilepsia 2017;58(7):1227-33.

4. Mohan RPS, Rastogi K, Bhushan R, Verma S. Phenytoin-induced gingival enlargement: a dental awakening for patients with epilepsy. BMJ Case Rep 2013;2013.

5. Weston J, Bromley R, Jackson CF, Adab N, ClaytonSmith J, Greenhalgh J, et al. Monotherapy treatment of epilepsy in pregnancy: congenital malformation outcomes in the child. Cochrane Database Syst Rev 2016;11:CDo10224.

6. Bentué-Ferrer D, Verdier MC, Tribut O. Pharmacological therapeutic follow-up of primidone and phenobarbital. Therapie 2012;67 (4):381-90.

7. Zaccara G, Perucca E. Interactions between antiepileptic drugs, and between antiepileptic drugs and other drugs. Epileptic Disord 2014;(4):409-31.

8. Patsalos PN, Berry DJ, Bourgeois BFD, Cloyd JC, Glauser TA, Johannessen SI, et al. Antiepileptic drugs best practice guidelines for therapeutic drug monitoring: a position paper by the sub commission on therapeutic drug monitoring, ILAE Commission on Therapeutic Strategies. Epilepsia 2008;49(7):1239-76.

9. Brodie MJ, Besag F, Ettinger AB, MulaM, Gobbi G, Comai S, et al. Epilepsy, antiepileptic drugs, and aggression: an evidence based review. Pharmacol Rev 2016;68(3):563-602.

10. Hamed SA. The auditory and vestibular toxicities induced by antiepileptic drugs. Expert Opin Drug Saf 2017;16(11):1281-94.

11. Tateno A, Sawada K, Takahashi I, Hujiwara Y. Carbamazepine- induced transient auditory pitchperception deficit. Pediatr Neurol 2006;35(2):131-4.

12. Gur-Ozmen S, Nirmalananthan N, von Oertzen TJ. Change of pitch due to carbamazepine and oxcarbazepine independently. Seizure 2013;22(2):162-3.

13. Maguire MJ. Music and epilepsy: a critical review: music and epilepsy. Epilepsia 2012;53(6):947-61. 
14. Kawai M, Kanemoto K. A case of auditory disturbance caused by lacosamide. Epilepsy Behav Case Rep 2019;11:70-2.

Cite this article as:

Bhasker A. Antiepileptics and Sound Perception Disorders. Int Healthc Res

Source of support: Nil, Conflict of interest: None declared

J. 2020;4(2):28-30. https://doi.org/10.26440/IHRJ/0402.05347

AUTHOR AFFILIATIONS: ( ${ }^{*}$ Corresponding Author) MBBS, Private Practitioner, Parwanoo, Himachal Pradesh

Corresponding Address:

Dr. Ashwani Bhasker

H No. 109, Sector 1

Parwanoo

Himachal Pradesh -173220
Contact corresponding author at: ashwanibhasker61[at]gmail[dot]com 